\title{
A Context-Aware Machine-to-Machine-Enabled Pervasive Cardiac Telemetry for Personalizing Health Care Delivery
}

\author{
Aude Aude-Elvis ODELOUI aeodeloui@gouv.bj \\ Thierry Edoh oscar.edoh@gmail.com
}

\begin{abstract}
Personalized health care in coming and shows promise to improve the health care services delivery. Internet of Things (IoT) enabled personalized care enables patient-centric care delivery where medical doctors provide care based on data emanated from the patient and on the patient medical/health record.

Cardiovascular diseases, known as non-communicable diseases, are a leading death cause worldwide. Developing countries are bearing the heaviest heart diseases burden. Additionally, these countries are facing numerous challenges such as poor access to health care services delivery, lack of adequate medicine, lack of health insurance that aggravate the poor care accessibility issues. Several outpatients living in rural are severely facing the accessibility issues due to the remote residence. They are living very far from specialized hospitals or clinics.
\end{abstract}

This paper aims at presenting a novel cardiac telemetry approach combining the paradigm of context awareness and the IoT Technology to provide personalized care to remote outpatient and, thus, improve on one hand the patient monitoring and on other increase access to health care services as well as provide personalized care, which shows promise to improve the quality of the care.

Regarding the telecommunication issues facing most developing, especially the rural regions, store and forward approach is adopted for data exchanges and machine-to-machine (M2M) communication is selected to support the communication in the entire system since M2M communication is technology independent, interoperable, and enable remote communication.

Keywords Patient telemonitoring, Machine-to-Machine, Patient-centric Care, Patient-Centered Care, Internet of Health Things, Device-to-Device, Data Analytics, Telemetry, Telecardiology, Mobile cardiovascular 